\section{Research Square}

Preprints are preliminary reports that have not undergone peer review.

They should not be considered conclusive, used to inform clinical practice, or referenced by the media as validated information.

\title{
Expression of Sortilin in Pancreatic Carcinoma and Influence of Pancreatic Cancer Cells by Sortilin siRNA Knockdown
}

\author{
Di Xu ( $\nabla$ xudi0323@nsmc.edu.cn) \\ North Sichuan Medical College \\ Fang Yu \\ North Sichuan Medical College \\ shangqing Liu \\ North Sichuan Medical College \\ Rong Cai \\ North Sichuan Medical College
}

Article

Keywords: Pancreatic cancer, sortilin, proliferation, invasion, migration

Posted Date: April 6th, 2022

DOI: https://doi.org/10.21203/rs.3.rs-1007352/v3

License: @ (i) This work is licensed under a Creative Commons Attribution 4.0 International License. Read Full License 


\section{Abstract}

Background: Currently, there are few effective therapeutic options for pancreatic cancer patients.Sortilin is a member of vps10p receptor family खreported in many types of cancers. However, the underlying mechanism and prognostic value of sortilin in pancreatic cancer are still unclear.

Objective:Understand the expression of sortilin in pancreatic cancer, and analyze its mechanism that affects the occurrence and development of pancreatic cancer.

Methods:A tissue microarray of 115 pancreatic cancer metastases was analyzed by immunohistochemistry. All data were analyzed by univariate analysis and multivariate analysis.Multivariate logistic regression analysis and the area under the receiver operating characteristic (ROC) curve were used to analyze the ability of sortilin in predicting pancreatic cancer.Next, survival analysis was performed to compare the survival time between high-risk and low-risk patients to validate the prognosis prediction efficacy of sortilin. The effects of sortilin on the invasion $\searrow$ metastasis and proliferation of pancreatic cancer cells were investigated both in vitro. investigate the anti-cancer effect of soritlin on human pancreatic cancer Capna1 and Bxpc3 cells, and its possible molecular mechanism.

Results:Differential expression analysis of 115 tissue microarrays showedsortilin expression was up-regulated in pancreatic cancer tissues, and it mainly comes from the nucleus. Sortilin expression in nucleus(SEIN) was only negatively correlated with $\mathrm{N}$ stage, Binary logistic regression model showed that SEIN is a good diagnostic marker for predicting pancreatic cancer and the accuracy of prediction is as high as $79.1 \%$. ROC curve analysis demonstrated a statistically significant diagnostic value of SEIN,and the diagnostic accuracy was $86.3 \%$, The Youden Index was calculated to evaluate the diagnostic power, The cut-off value for SEIN in pancreatic cancer diagnosis was 0.85 , with a sensitivity of $90.9 \%$ and a specificity of $68.3 \%$. Univariate analysis showed that $M$ stage $(P=0.022)$, histological grade $(P=0.021)$, clinic stage $(P=0.030)$ and $\operatorname{SEIN}(P=0.039)$ were correlated with prognosis of pancreatic cancer patients, Multivariate regression analysis showed that $\mathrm{M}$ stage $(P=0.036)$ and $\operatorname{ESIN}(P=0.004)$ were independent factors.

The proliferation, invasion and migration of pancreatic cancer cells were inhibited in vitro by sortilin siRNA knockdown.it may have something to do with soritlin/P53/NFKB regulated the the proliferation function and sortilin/MMP9 regulated the invasion-promotion of pancreatic cancer cells.

Conclusions: These fndings demonstrated that the low expression of SEIN indicates better prognosis in pancreatic cancer and supplemented the effect mechanism of sortilin on pancreatic cancer cells. SEIN expression may serve as a potential diagnostic indicator of pancreatic cancer.

\section{Introduction}

Pancreatic cancer(PC) remains one of the most lethal types of cancer.It is the 11th most common cancer worldwide. There are no current screening recommendations for pancreatic cancer; thus primary prevention is of utmost importance[1].More than $90 \%$ of pancreatic cancers are ductal adenocarcinoma and its variants[2].It has been reported that inactivated mutations of tumor suppressor genes (such as CDKN2A / p16, TP53 and Smad4) and KRAS mutations can cause the growth of invasive PDAC tumors[3], which is far from meeting the clinical needs.

Neurotrophic factor family (NTs) not only plays an important role in regulating the strength, number of synaptic connections and neurotransmission, but also has a good performance in cancer[4]. Sortilin is a member of NTS factor family receptors and has been reported in a number of cancers such as: human adenocarcinoma epithelial cell line (HT29) [5], breast cancer[6], lung cancer A549 cells[7] and prostate cancer cells. But it is rare in pancreatic cancer.

In this study, we focused on the role of sortilin in pancreatic cancer and explored its relationship with the pathogenesis of pancreatic cancer.

\section{Materials And Methods}

Immunohistochemistry(IHC) 
The PC tissue microarray (hpan-ade120sur-01) consisted of 115 points in 60 cases were bought from Shanghai core super Biotechnology Co., Ltd.A full description of the prototype IHC assay is provided in the supplementary methods of Akturk $\mathrm{G}$ et al.[9]. In brief, the unstained slides of tissue microarray were first baked at $60^{\circ} \mathrm{C}$, deparaffinized in xylene, and rehydrated through a series of graded ethanols into distilled water. Antigens in the tissue were unmasked by placing the slides into a steamer withcitrate buffer , the slides were incubated overnight with the primary antibody (human sortilin polyclonal goat IgG antibody, AF3154, R\&D Biotechnology) diluted in DAKO Primary Antibody Diluent $(2 \mu \mathrm{g} / \mathrm{mL}$ ). After rewarming, add the secondary antibody (substance-labeled rabbit antigoat).Detection of the primary antibody was performed using DAB reagents (horseradish peroxidase polymer, diaminobenzidine chromogen, and diaminobenzidine enhancer), washing with PBS between the incubation steps. The slides were counterstained with hematoxylin, coverslipped and viewed with the aid of a light microscope.

The interpretation is according to TPS[10]. TPS=(Thenumberof viable tumor cells positive for sortilin/total number of viable neoplasti ccells) $\times 100 \%$. 0 points (negative), 1 point (1-25\%), 2 points (26\%-50\%), 3 points $(51-75 \%), 4$ points $(76 \%-100 \%)$, take the median( $90 \%)$ as the standard,and score less or equal than $90 \%$ was defined as low expression, and more than $90 \%$ as high expression.

\section{Cell culture}

The pancreatic cancer cells, Capan1 and Bxpc3, are from the Imaging Laboratory of North Sichuan Medical College. Capan1 cells were grown in IMDM (SH30228.01, Hyclone) with 20\% FBS. Bxpc3 cells were cultured in RPMI-1640 Medium (SH30809.01, Hyclone), supplemented with $10 \%$ fetal bovine serum (11011-8611, Sijiqing, Zhejiang Tianhang Biotechnology Co., Ltd.) at $37^{\circ} \mathrm{C}$ in a $5 \% \mathrm{CO}_{2}$ incubator.Cells were dissociated with $0.25 \%$ Trypsin-EDTA (PYG0015, Boster Biological Technology co.Itd) at $37^{\circ} \mathrm{C}$ for $5 \mathrm{~min}$ and collected by centrifugation at $1000 \mathrm{rpm}$ for $5 \mathrm{~min}$. Cells were resuspended in a new complete medium and placed in an incubator for further culture.

\section{Cell siRNA transfection}

The Capan 1 and Bxpc3 cells were seeded in 6-well plates at $50 \%$ confluence 1 day prior to transfection. We used a ratio of $50 \mathrm{pmol}$ siRNA (SR304211,OriGeneTechnologies) Rfect 10 $\mathrm{\mu l}(11013$, Changzhou Baidai Biotechnology Co., Ltd) to mix them in $500 \mu \mathrm{l}$ of serumfree medium for $20 \mathrm{~min}$ and then the siRNA/Rfect mixture was added to the cells with $2 \mathrm{ml}$ of complete medium overnight. The targets equences for siRNA are siRNA-A: GCAGAGCUAGAUAUAGCAC, siRNA-B: CGCAAGGACAGGGUACAAACUUAGC, siRNA-C: AGACGUAGGAAACUCAAUAUUCUTC. Transfection of cells were performed in triplicate. The transfection effect of sortilin knockdown was evaluated by RT-PCR and verified by western blot.

\section{Reverse Transcription Polymerase Chain Reaction(RT-PCR)}

Cells were collected at 24hours (Bxpc3) and 48 hours (Canpan-1) after transfection. The cells in a well of 6-well plate were mixed with $1 \mathrm{~mL}$ of TRIzol(DP405-02, Tiangen Biotech (Beijing) Co.,Ltd.). Total RNA was extracted according to the manufacturer's instructions using a Total RNA Extraction Kit(TR201-100, Beijing Tianmo Technology Development Co., Ltd.). Total RNA concentration and OD value were measured, and reverse transcribed into cDNA by Reverse Transcription Kit (K1622, Thermo Fisher Scientific). The qPCR reactions ( $20 \mu \mathrm{l}$ final volume) were conducted using the Bestar ${ }^{\circledR}$ SybrGreen qPCR Mastermix (DBI-2043,DBI®Bioscience). See Table I for primer sequence.Reaction conditions for RT-PCR were denaturation for $5 \mathrm{~min}$ at $95{ }^{\circ} \mathrm{C}, 95{ }^{\circ} \mathrm{C}$ for $30 \mathrm{sec}, 55^{\circ} \mathrm{C}$ for $30 \mathrm{sec}$, and 72 ${ }^{\circ} \mathrm{C}$ for $40 \mathrm{sec}$ for $35 \mathrm{cycles} .2^{-\Delta \Delta \mathrm{ct}}$ were used to analyze the results. Each experiment was repeated three times.

\section{Cell scratch test}

A marker pen was used to draw an uniform line every $0.5-1 \mathrm{~cm}$ at the back of the 6-well plate, with three transverse lines crossed over each well. After 24h transfection, Capan1and Bxpc3 cells were overpaved to 12-well plates. Three horizontal lines were drawn perpendicular to the reference line. The cells were washed three times with PBS and added serum-free medium, at $37^{\circ} \mathrm{C}$ with $5 \% \mathrm{CO}_{2}$ for 24 hours.Samples were taken at $\mathrm{t}=0,12 \mathrm{hours}$. Each result was repeated three times.

\section{CCK8 cell proliferation experiment}

Cells were plated on 96 well plates at concentration of 5000 cells/well. The cells were cultured in an incubator at $37^{\circ} \mathrm{C} 5 \% \mathrm{CO}_{2}$ for 24

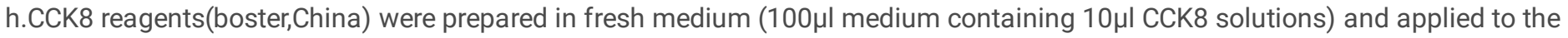


cells.After cell treatments, the incubation was continued for 2 hours at $37^{\circ} \mathrm{C} 5 \% \mathrm{CO}_{2}$.Absorbance was measured at $450 / 540 \mathrm{~nm}$ (Sunrise ${ }^{\text {TM }}$ Absorbance Reader).Each result was repeated three times.

\section{Transwell cell invasion experiment}

Matrigel (BD Biosciences, Beijing, China) was thawed overnight at $4^{\circ} \mathrm{C}$ and then kept on ice.Matrigel was diluted by serum-free medium ( $1: 8$ dilution) on ice and added $45 \mu \mathrm{l}$ to transwell chambes for $2 \mathrm{~h}$ at $37^{\circ} \mathrm{C}$. The transfected cells $\left(20 \times 10^{4}\right)$ in $100 \mu \mathrm{l}$ were added to the upper chamber of the transwell chamber, and $600 \mu \mathrm{l}$ of medium containing $20 \%$ fetal bovine serum was added to the lower chamber at $37^{\circ} \mathrm{C} 5 \% \mathrm{CO}_{2}$ for $72 \mathrm{hours}$. The lower chamber was washed with PBS for three times, following which a cotton bud was used to remove cells and medium from the upper chambers,fixed with methanol for 15 min, washed with PBS and stained with Giemsa solution (Gibco BRL) for 30 min. The stained cells were photographed with a digital camera. The number of colonies in each well was counted with Image $\mathrm{J}$ software.Each result was repeated three times.

\section{Western blot experiment}

MIP-3a(10485-H07E, Sino Biological Solution Specialist)[11, 12]was added to the culture medium at a final concentration of $100 \mathrm{ng} / \mathrm{ml}$, incubating for $24 \mathrm{~h}$. After $48-72 \mathrm{~h}$ of transfection, the medium was discarded and 100 $\mu$ l of RIPA lysate (P0013B, Beyotime Biotechnology) was added into each well.After 48 hours transfection, $2 \mu \mathrm{g} / \mathrm{ml}$ puromycinwas added into the medium. The primary antibodies MMP9(13667, Cell Signaling Technology), p53(2527, Cell Signaling Technology), NFkB p65(8242, Cell Signaling Technology) are rabbit monoclonal antibodies, and Sortilin (AF3154,R囚D Bio-techne brand) is Antigen Affinity-purified Polyclonal Goat IgG. The secondary antibody uses HRP Conjugated AffiniPure Goat Anti-rabbit IgG(BA1054囚Boster Biological technology Co., Ltd.) and HRP Conjugated AffiniPure Rabbit Anti-goat IgG(BA1060هBoster Biological technology Co., Ltd.).The test refers to the steps summarized by Sean $\mathrm{C}$ Taylor et al. The relative gray scale of the bands was analyzed using Image $\mathrm{J}$ software.Each result was repeated three times.

\section{Statistical analysis}

Statistical analysis was performed using SPSS23.0 and Graphpad prism7 software.Measurement data was presented as mean \pm standard deviation (SD).Enumeration data and categorical variables were analyzed using $c^{2}$ or Fisher's exact tests. The median survival time and the mean survival time were calculated by Kaplan-Meier method. No significant $(P>0.05)$, Statistically significant difference was considered at *:P<0.05, ${ }^{\star *}: P<0.01,{ }^{\star * *}: P<0.001$ and $* \star \star *: P<0.0001$ between groups.

The Receiver Operating Characteristic (ROC) curve and binary logistic regression were applied to IHC data.

\section{Results}

\section{Differentexpressions of sortilin in pancreatic cancer and adjacent tissues}

Firstly, the online database GEPIA was used to investigate sortilin different expressions between different cancer and normal tissues(Figure1A).Including pancreatic cancer, the content of sortilin in pancreatic cancer was significantly higher than that in adjacent tissues(Figure1B).

After immunohistochemical staining of 115 pancreatic cancer tissue chips (Table II,Figure2), sortilin is highly expressed in the nucleus of pancreatic cancer tissues(20\%), and almoste not in adjacent normal tissue( $0 \%$. The difference was highly significant $(P<0.0001)$. Sortilin are both highly expressed in the cytoplasm of pancreatic cancer tissues( $98 \%)$ and adjacent normal tissue( $95 \%)$. The difference was not significant $(P=0.897)$.

\section{Binary Logistic Regression (BLR)model for the estimation of Prediction of pancreatic cancer from SEIN}

BLR modeling was used to predict pancreatic cancer.The diagnostic accuracy is $73.9 \%$.Moreover, the ROC curve analysis showed that SEIN had good diagnostic value for PC(Figure 3). The cutoff point of 0.85 , calculated by the Youden Index, had $90.9 \%$ sensitivity and $68.3 \%$ specificity (AUC $=0.863,95 \% \mathrm{Cl} 0.852-0.922)$.

\section{Correlation between SEIN and clinicopathological features of 60 PC patients.}


In view of the up regulation of SEIN, we further analyzed the relationship between SEIN in 60 pancreatic cancer tissues and various

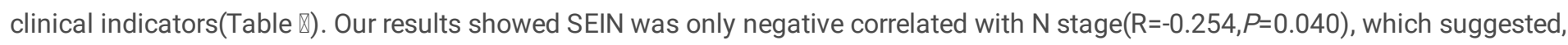
SEIN is related to lymphatic metastasis of pancreatic cancer.

Association of clinicopathological indicators and prognostic factors. Univariate survival analyses indicated that pathological grade,SEIN, M stage, and clinical stage were associated with prognosis, while gender, age, tumor size, $\mathrm{T}$ stage, and $\mathrm{N}$ stage were not

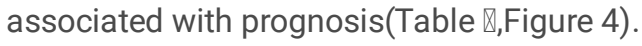

To determine the independent prognostic factors, we performed a multivariate analysis on the statistically significant factors in the univariate analysis. It is suggested that $\operatorname{ESIN}(P=0.004)$ and $\mathrm{M}$ stage $(P=0.0 .36)$ could be used as an independent prognostication factor for PC patients, but the pathological grade $(P=0.248)$ and clinical stage $(P=0.560)$ could not be used as an independent factor for the

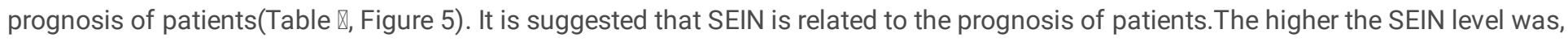
the higher the risk of death was.

\section{Validation of siRNA knockdown of an effective fragment of sortilin from mRNA level and protein level}

Cells were divided into five groups (blank control group,negative control group,siRNA-A group,siRNA-B group, and siRNA-C group).Capan 1 cells and Bxpc3 cells were collected afte48 and 24 hoursr transfection respectively,then knockdown fragment were screened. It was identified that siRNA-B and siRNA-C had knockdown effect on the sortilin of both cells, while siRNA-A had no knock down effect from the mRNA level(Table $\mathbb{\Delta}$ Figure 6).

\section{Migration of Pancreatic cancer cells after soritlin knockdown}

Aafter 12 hours transfection, the cells in blank group, negative group and mock group showed different degree of migration, but there was no significant migration in transfection group. It is suggested that the inhibition of sortilin can effectively control the migration of pancreatic cancer cells(Figure 7).

\section{Proliferation of pancreatic cancer cells after sortilin knockdown}

After transfection, cells were collected at different time points. After 48 hours transfection, the proliferation of capan 1 cells decreased in knockdown group, with a inhibition rate of $71.21 \%$. After 24 hours transfection, the proliferation of Bxpc3 cells decreased in knockdown group, with a inhibition rate of $52.28 \%$. The OD values of the two cells in each time period were shown in Table区 and Figure 8.After sotilin was inhibited, the proliferation ability of two cell lines decreased at different time, suggesting that sortilin is related to the proliferation of pancreatic cancer cells, which can be inhibited by intervention of sotilin.

\section{Invasion ability of pancreatic cancer cells after sortilin knockdown}

Our data showed that after sotilin inhibition, the invasiveness of the two cells in the inhibition group (si) was significantly lower than that in the Blank group. It is suggested that the inhibition of sortilin can effectively reduce the invasiveness of pancreatic cancer cells(Figure 9).

\section{After sortilin inhibition】mRNA expression of P53, NFKB and MMP9}

After siRNA transfection, the expression of P53, NFKB and MMP9 mRNA was detected. NFKB and MMP9 decreased, while P53 increased. There was no significant difference between blank group, negative control group and mock group,The blank group and knockdown group were statistically significant difference(Table $\otimes$ and Table $\otimes$, Figure 10).It is suggested that sortilin may affect the migration, invasion and proliferation of pancreatic cancer cells in relation to P53, NFKB and MMP9. The inhibition of sortilin may affect the migration, invasion and proliferation of pancreatic cancer cells through the increase ofP53 and the decrease of NFKB and MMP9.

\section{Disscussion}

Pancreatic cancer is one of the pancreatic cancers with very poor prognosis. The main risk factors of pancreatic cancer include smoking, obesity, long-standing diabetes and family history of disease.At the same time, people still have insufficient knowledge about 
the development of pancreatic cancer[13].So, it is still important to explore the mechanism of PC and find new biomarkers at an early stage.

In this study, The expression of sortilin in various cancers was retrieved using the bioinformatics website GEPIA, including pancreatic cancer tissues among them. Sortilin was indeed up-regulation in 115 cases of pancreatic cancer tissues which was verified by IHC. This is consistent with the report by Fangfang Gao et al[14]. In this study, we analyzedfor the first time that the up-regulation of soritlin comes from SEIN. We continue to analyze the results of IHC in depth. The predictive ability of sortilin was evaluated by BLR and ROC curve, The predictive value of SEIN was $73.9 \%$, and the diagnostic accuracy was $86.3 \%$. The Youden Index was calculated to evaluate the diagnostic power. The cut-off value of SEIN for PC diagnosis was 0.85 , with the corresponding sensitivity of $90.9 \%$ and the specificity of $68.3 \%$. Univariate Survival analysis showed that the expression level of SEIN, M stage, Pathological grade and Clinical stage had a significant impact on the survival time, and patients with higher expression level of SEIN had poor prognosis. Multivariate analysis suggested that SEIN might be an independent prognostic indicator for the survival of PC patients.

Based on IHC research results we further verified the role of sortilin in the development of PC through in vitro. After we specifically knocked down sortilin with small interfering siRNA fragments, both pancreatic cancer cells showed reduced proliferation, invasion and migration.This observation is consistent with the results from IHC characterization. Target reduction of sortilin is somewhat helpful for controlling the progression of pancreatic cancer and improving the survival rate of PC patients.

This study also further analyzed the mechanism of sortilin in controlling the proliferation, migration and invasion of pancreatic cancer cells. We introduced P53, NFKB and MMP9. It was found that p53 was up-regulated, and NFKB and MMP9 in a differen degress of down-regulated after reduction of sortilin. We preliminarily estimated that the decrease of sortilin may be caused by sortilin/NFKB/p53 pathway affecting cell proliferation and sortilin-MMP9 pathway affecting cell invasion. A large number of reports have pointed out that sortilin binds to different ligands and has different effects on cells. When it is combined with ProNGF, it induces tumor cell apoptosis and arterial remodeling[15]; high affinity binding with ProNGF and p75NTR complex, mediates cell death of central neurons, natural killer cells, and retinal photoreceptors[16] and activates substantia nigra Caspase-mediated dopamine neuronal death signaling pathway in the striatum[17]. Binding to the p75NTR/TrkB complex plays a key role in cancer cell survival [18]. Most reports are about the close association between sortilin and neurotrophic factor family. We have opened a new way for sortilin to act in pancreatic cancer, but more data is still needed to support, also it is necessary to further expand the analysis that whether it has similar performance in other cell lines of PC.

\section{Abbreviations}

PC

Pancreatic cancer

ROC

the receiver operating characteristic

SEIN

Sortilin expression in nucleus

NTs

Neurotrophic factor family

$\min$

minute

$\mathrm{sec}$

second

BLR

Binary Logistic Regression

siRNA

small interference RNA

IHC

Immunohistochemistry

RT-PCR

Reverse Transcription Polymerase Chain Reaction. 


\section{Declarations}

\section{Acknowledgements}

We want to thank acknowledge the database available to us for this study.

\section{Ethics approval and consent to participate}

This study was approved by the Ethics Committee of North sichuan Medical college (Nanchong, China) in accordance with the principles of the Declaration of Helsinki.

The patient's informed consent was obtained and their medical data wereused for research.

\section{Consent for publication}

All authors have seen and agreed to publish.

\section{Competing interests}

The authors declare that they have no known competing financial interests or personal relationships that could have appeared to influence the work reported in this paper.

\section{Author details}

1 The first Corresponding author. Master of Human Anatomy, Histology and Embryology, North Sichuan Medical College, 2017, Nanchong.Clinical Laboratory,Public health clinical center of chengdu,637000, PR China,xudi0323@nsmc.edu.cn.

2 Master of Human Anatomy, Histology and Embryology, North Sichuan Medical College, 2019, Nanchong, 637000, PR China,yufang217815@nsmc.edu.cn.

3 Department of Human Anatomy, North Sichuan Medical College, Nanchong, 637000, PR China,liushangqing0913@nsmc.edu.cn. 4 The second Correspondence author.School of Nursing, North Sichuan Medical College, Nanchong, 637000, PR China. E-mail addresses: cairong1206@nsmc.edu.cn.

\section{Funding}

This work was supported by 2015 Research and Development Fund Project of North Sichuan Medical College (CBY15-A-YB04), Sichuan Provincial Department of Human Resources and Social Security 2014 Preferential Funding Project for Scientific and Technological Activities of Overseas Returnees (2014167-10), Sichuan Provincial Department of Education 2014 Key Project (14ZA0181)and Special Fund Project of Nanchong City-University Cooperation in 2017 (NSMC20170409).

\section{Authors' contributions}

CONCEPTION:DX designed the work and wrote the manuscript.

INTERPRETATION OR ANALYSIS OF DATA:DX is mainly responsible for interpretation and analysis of Data;FY,RC, and SQL participated in data analysis andthe discussion and language editing.

PREPARATION OF THE MANUSCRIPT:DXis mainly responsible for preparation of the manuscript,FY,RC and SQL collected clinical information.

REVISION FOR IMPORTANT INTELLECTUAL CONTENT:RC and SQL reviewed the manuscript.

SUPERVISION:RC and SQL are responsible for the supervision.

\section{Availability of data and materials}


The datasets supporting the conclusions of Figure 1 are available in囚[GEPIA dataset] at (http://gepia.cancer-pku.cn/detail.php? gene=SORT1).Please contact the author for the rest of the data.

\section{References}

1. Ilic, M. and I. Ilic, Epidemiology of pancreatic cancer. World J Gastroenterol, 2016. 22(44): p. 9694-9705.

2. Wang, C.A., et al., DUSP2 regulates extracellular vesicle-VEGF-C secretion and pancreatic cancer early dissemination. J Extracell Vesicles, 2020. 9(1): p. 1746529.

3. Grant, T.J., K. Hua and A. Singh, Molecular Pathogenesis of Pancreatic Cancer. Prog Mol Biol Transl Sci, 2016. 144: p. 241-275.

4. Jimenez-Luna, C., et al., Proteomic biomarkers in body fluids associated with pancreatic cancer. Oncotarget, 2018. 9(23): p. $16573-16587$.

5. Massa, F., et al., Focal adhesion kinase dependent activation of the PI3 kinase pathway by the functional soluble form of neurotensin receptor-3 in HT29 cells. Int J Biochem Cell Biol, 2013. 45(5): p. 952-9.

6. Rhost, S., et al., Sortilin inhibition limits secretion-induced progranulin-dependent breast cancer progression and cancer stem cell expansion. Breast Cancer Res, 2018. 20(1): p. 137.

7. Al-Akhrass, H., et al., Sortilin limits EGFR signaling by promoting its internalization in lung cancer. Nat Commun, 2017. 8(1): p. 1182.

8. Tanimoto, R., et al., Sortilin regulates progranulin action in castration-resistant prostate cancer cells. Endocrinology, 2015. 156(1): p. $58-70$.

9. Akturk, G., et al., Multiplexed Immunohistochemical Consecutive Staining on Single Slide (MICSSS): Multiplexed Chromogenic IHC Assay for High-Dimensional Tissue Analysis. Methods Mol Biol, 2020. 2055: p. 497-519.

10. Dolled-Filhart, M., et al., Development of a Companion Diagnostic for Pembrolizumab in Non-Small Cell Lung Cancer Using Immunohistochemistry for Programmed Death Ligand-1. Arch Pathol Lab Med, 2016. 140(11): p. 1243-1249.

11. Campbell, A.S., et al., Macrophage inflammatory protein-3alpha promotes pancreatic cancer cell invasion. J Surg Res, 2005. 123(1): p. 96-101.

12. Kimsey, T.F., et al., Co-localization of macrophage inflammatory protein-3alpha (Mip-3alpha) and its receptor, CCR6, promotes pancreatic cancer cell invasion. Cancer J, 2004. 10(6): p. 374-80.

13. Drouillard, A., et al., [Epidemiology of pancreatic cancer]. Bull Cancer, 2018. 105(1): p. 63-69.

14. Gao, F., et al., The Membrane Protein Sortilin Can Be Targeted to Inhibit Pancreatic Cancer Cell Invasion. Am J Pathol, 2020. 190(9): p. 1931-1942.

15. Blondy, S., et al., Neurotrophins and their involvement in digestive cancers. Cell Death Dis, 2019. 10(2): p. 123.

16. Nakamura, K., et al., Intracellular sortilin expression pattern regulates proNGF-induced naturally occurring cell death during development. Cell Death Differ, 2007. 14(8): p. 1552-4.

17. Wang, T., et al., Protective effects of octacosanol on 6-hydroxydopamine-induced Parkinsonism in rats via regulation of ProNGF and NGF signaling. Acta Pharmacol Sin, 2010. 31(7): p. 765-74.

18. Béraud-Dufour, S., et al., Focal Adhesion Kinase-Dependent Role of the Soluble Form of Neurotensin Receptor-3/Sortilin in Colorectal Cancer Cell Dissociation. Int J Mol Sci, 2016. 17(11).

\section{Tables}

\section{Table I. Primer sequence of related molecule}




\begin{tabular}{|lllll|}
\hline \multirow{2}{*}{ sortilin } & Primer sequence & Base number & $\mathrm{Tm}$ & Productlocatin \\
& Up stream: aagtctttggaccgacatctct & 22 & 60.8 & $751-772$ \\
& Down stream: agcacgcttgttatgtagacg & 21 & 60.4 & $845-825$ \\
& & & & \\
\hline \multirow{2}{*}{ GAPDH } & Up stream: ggagcgatcctccaaaaat & 21 & 61.6 & $108-128$ \\
& Down stream: ggctgtcatacttcatgg & 23 & 60.9 & $304-282$ \\
\hline \multirow{2}{*}{ P53 } & Up stream: cagcacatgacggaggttgt & 20 & 62.4 & $382-401$ \\
& Down stream:tcatccaaaaaactactccacgc & 21 & 60.1 & $506-486$ \\
\hline \multirow{2}{*}{ NFKB } & Up stream: aacagagaggatttcgtccg & 22 & 60.3 & $155-176$ \\
& Down stream: tttgacctgaggtaagacttct & 23 & 60.4 & $258-236$ \\
\hline \multirow{2}{*}{ MMP9 } & Up stream: agacctggggattccaaac & 21 & 62.0 & $306-326$ \\
& Down stream: cggcaagtcttccgagtagt & 20 & 61.3 & $399-380$ \\
\hline
\end{tabular}

Table II. Differential expression of sortilin in pancreatic cancer and its adjacent tissues

\begin{tabular}{lllllll} 
& High expression & Low expression & Total & Positive rate & Mean \pm Std. Deviation & $P$ value \\
\cline { 1 - 3 } cancer cytoplasm & 59 & 1 & 60 & $98 \%$ & $4.867 \pm 1.295$ & 0.897 \\
\cline { 1 - 3 } & 52 & 3 & 55 & $95 \%$ & $4.848 \pm 1.152$ & \\
\cline { 1 - 2 } $\begin{array}{l}\text { cancer } \\
\text { nucleus }\end{array}$ & 12 & 48 & 60 & $20 \%$ & $6.158 \pm 1.345$ & 0.001 \\
\cline { 1 - 2 } & 0 & 55 & 55 & $0 \%$ & $3.679 \pm 1.181$ &
\end{tabular}

Table \. Correlation between SEIN and clinicopathological features of $60 \mathrm{PC}$ patients 


\begin{tabular}{|c|c|c|c|c|c|}
\hline & $\mathrm{N}$ & $\mathrm{L}$ & $\mathrm{H}$ & $\mathrm{R}$ & $p$ \\
\hline \multicolumn{6}{|l|}{ Age } \\
\hline$\leq 60$ & 24 & 18 & 6 & 0.179 & 0.076 \\
\hline$\nabla 60$ & 36 & 26 & 10 & & \\
\hline \multicolumn{6}{|l|}{ Sex } \\
\hline men & 35 & 25 & 10 & 0.070 & 0.559 \\
\hline women & 25 & 19 & 6 & & \\
\hline Tumor & & & & & \\
\hline$\leq 5 \mathrm{~cm}$ & 52 & 39 & 13 & 0.102 & 0.335 \\
\hline$\nabla 5 \mathrm{~cm}$ & 8 & 5 & 3 & & \\
\hline \multicolumn{6}{|c|}{$\begin{array}{l}\text { Pathological grade } \\
\text { stage }\end{array}$} \\
\hline$\nabla / \nabla$ & 31 & 21 & 10 & 0.171 & 0.157 \\
\hline$\square$ & 29 & 23 & 6 & & \\
\hline \multicolumn{6}{|c|}{ 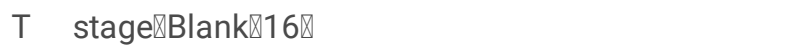 } \\
\hline $\mathrm{T} 1$ & 2 & 1 & 1 & 0.075 & 0.587 \\
\hline T2 & 6 & 4 & 2 & & \\
\hline T3 & 36 & 27 & 9 & & \\
\hline \multicolumn{6}{|l|}{$\mathrm{N}$ stage } \\
\hline NO & 33 & 23 & 10 & -0.254 & 0.040 \\
\hline N1 & 27 & 18 & 9 & & \\
\hline \multicolumn{6}{|l|}{ M stage } \\
\hline MO & 56 & 41 & 15 & -0.145 & 0.229 \\
\hline M1 & 4 & 3 & 1 & & \\
\hline \multicolumn{6}{|c|}{ Clinical stage $₫ B l a n k: 9 \rrbracket$} \\
\hline ( & 5 & 3 & 2 & -0.030 & 0.814 \\
\hline प & 42 & 31 & 11 & & \\
\hline ? & 4 & 3 & 1 & & \\
\hline
\end{tabular}

Table . Univariate analysis of prognostic factors 


\begin{tabular}{|c|c|c|c|c|c|c|c|}
\hline & & $\mathrm{T}(\mathrm{N})$ & Death & Survival & $\begin{array}{l}\text { Survival } \\
\text { rate }\end{array}$ & $c^{2}$ & $p$ \\
\hline \multirow[b]{2}{*}{ Sex } & men & 35 & 28 & 7 & $19.4 \%$ & 0.285 & 0.593 \\
\hline & women & 25 & 19 & 6 & $22.2 \%$ & & \\
\hline \multirow[b]{2}{*}{ Age } & $\leq 60$ & 16 & 13 & 3 & $18.8 \%$ & 0.810 & 0.368 \\
\hline & $>60$ & 20 & 16 & 4 & $20.0 \%$ & & \\
\hline \multirow{3}{*}{ Tumor size } & $<5$ & 20 & 18 & 2 & $10.0 \%$ & 1.566 & 0.211 \\
\hline & $>5$ & 9 & 7 & 2 & $22.2 \%$ & & \\
\hline & $\mathrm{T} 1$ & 2 & 2 & 0 & $0.0 \%$ & 0.388 & 0.824 \\
\hline \multirow{2}{*}{$\mathrm{T}$ stage } & T2 & 7 & 5 & 2 & $28.6 \%$ & & \\
\hline & T3 & 38 & 29 & 9 & $23.7 \%$ & & \\
\hline \multirow[t]{2}{*}{$\mathrm{N}$ stage } & NO & 35 & 25 & 10 & $28.6 \%$ & 1.175 & 0.278 \\
\hline & $\mathrm{N} 1$ & 25 & 22 & 3 & $12.0 \%$ & & \\
\hline \multirow[t]{2}{*}{ M stage } & MO & 59 & 46 & 13 & $22.0 \%$ & 5.229 & 0.022 \\
\hline & M1 & 4 & 4 & 0 & $0.0 \%$ & & \\
\hline \multirow[t]{2}{*}{ Pathological grade } & $\nabla / \nabla$ & 34 & 25 & 9 & $26.5 \%$ & 5.353 & 0.021 \\
\hline & प & 29 & 25 & 4 & $13.8 \%$ & & \\
\hline \multirow{3}{*}{ Clinical stage } & ( & 5 & 3 & 2 & $40.0 \%$ & 6.986 & 0.030 \\
\hline & प & 45 & 34 & 11 & $24.4 \%$ & & \\
\hline & ૫ & 4 & 4 & 0 & $0.0 \%$ & & \\
\hline \multirow[b]{2}{*}{ SEIN } & L & 44 & 37 & 7 & $15.9 \%$ & 4.242 & 0.039 \\
\hline & $\mathrm{H}$ & 16 & 10 & 6 & $37.5 \%$ & & \\
\hline
\end{tabular}

Table . Multivariate analysis of independent prognosticfanctors of PC

\begin{tabular}{|lll|}
\hline$P$ Hazard degree \\
\hline Pathological grade & 0.248 & -0.543 \\
\hline N stage & 0.131 & 0.556 \\
\hline ESIN & 0.004 & 1.126 \\
\hline M stage & 0.036 & 1.763 \\
\hline Clinical stage & 0.560 & 0.378 \\
\hline
\end{tabular}

Table . Relative mRNA expression of sortilin in each group after siRNA transfection 


\begin{tabular}{|c|c|c|c|c|c|c|c|}
\hline & & sortilin $₫ \mathrm{Ct} \rrbracket$ & GAPDH(Ct) & $2^{-\Delta \Delta c t}$ & $\mathrm{~F}$ & $P 1$ & $P 2$ \\
\hline \multirow[t]{5}{*}{ Capan1 } & Blank & $24.046 \pm 0.076$ & $17.563 \pm 0.308$ & $1.007 \pm 0.17$ & \multirow{5}{*}{19.661} & \multirow{5}{*}{0.0001} & \\
\hline & NC & $23.13 \pm 0.111$ & $16.708 \pm 0.23$ & $1.045 \pm 0.081$ & & & 0.764 \\
\hline & SiRNA-A & $23.22 \pm 0.191$ & $16.668 \pm 0.296$ & $0.971 \pm 0.237$ & & & 0.7697 \\
\hline & SiRNA-B & $23.08 \pm 0.181$ & $15.318 \pm 0.039$ & $0.421 \pm 0.099$ & & & 0.001 \\
\hline & SiRNA-C & $24.206 \pm 0.34$ & $15.493 \pm 0.378$ & $0.23 \pm 0.096$ & & & 0.0001 \\
\hline \multirow[t]{5}{*}{ Вxpc3 } & Blank & $23.954 \pm 1.295$ & $14.898 \pm 0.542$ & $1 \pm 0.061$ & \multirow{5}{*}{26.932} & \multirow{5}{*}{0.0001} & \\
\hline & NC & $23.863 \pm 0.802$ & $15.007 \pm 0.222$ & $1.078 \pm 0.136$ & & & 0.441 \\
\hline & SiRNA-A & $30.914 \pm 7.518$ & $22.076 \pm 8.119$ & $1.096 \pm 0.17$ & & & 0.344 \\
\hline & siRNA-B & $26.674 \pm 0.661$ & $15.758 \pm 0.202$ & $0.341 \pm 0.257$ & & & 0.0001 \\
\hline & SiRNA-C & $25.129 \pm 1.418$ & $14.915 \pm 0.234$ & $0.453 \pm 0.18$ & & & 0.0001 \\
\hline
\end{tabular}

F:Global analysis of variance

$p 1: P$ value of global analysis of variance

p2:NC,siRNA-A,siRNA-B,siRNA-C vs Blank.

Table . The OD of two cell lines after knockdown sortilin in each time period

\begin{tabular}{|c|c|c|c|c|c|c|c|c|c|c|}
\hline & $\mathrm{T}$ & Blank & Mock & $\mathrm{NC}$ & si & $\mathrm{F}$ & P1 & $P 2$ & P3 & $\begin{array}{l}\text { Inhibition } \\
\text { rate }\end{array}$ \\
\hline \multirow[t]{3}{*}{ Capan1 } & $48 \mathrm{~h}$ & $1.041 \pm 0.126$ & $0.973 \pm 0.199$ & $0.985 \pm 0.193$ & $0.558 \pm 0.066$ & 20.653 & 0.136 & 0.64 & 0.0001 & $71.21 \%$ \\
\hline & $72 \mathrm{~h}$ & $0.913 \pm 0.104$ & $0.959 \pm 0.017$ & $0.955 \pm 0.053$ & $0.507 \pm 0.046$ & 164.01 & 0.982 & 0.931 & 0.0001 & $90.97 \%$ \\
\hline & $96 \mathrm{~h}$ & $1.008 \pm 0.222$ & $1.011 \pm 0.365$ & $0.989 \pm 0.147$ & $0.369 \pm 0.094$ & 11.256 & 0.987 & 0.978 & 0.0001 & $73.39 \%$ \\
\hline \multirow[t]{3}{*}{ Вxpc3 } & $24 \mathrm{~h}$ & $1.194 \pm 0.102$ & $1.058 \pm 0.092$ & $1.104 \pm 0.061$ & $1.068 \pm 0.088$ & 58.894 & 0.096 & 0.505 & 0.0001 & $52.28 \%$ \\
\hline & $48 \mathrm{~h}$ & $1.088 \pm 0.104$ & $1.058 \pm 0.118$ & $1.135 \pm 0.133$ & $0.32 \pm 0.154$ & 65.398 & 0.859 & 0.39 & 0.0001 & $80.89 \%$ \\
\hline & $72 \mathrm{~h}$ & $1.156 \pm 0.14$ & $1.098 \pm 0.14$ & $1.109 \pm 0.198$ & $0.3 \pm 0.191$ & 44.806 & 0.531 & 0.623 & 0.0001 & $88.00 \%$ \\
\hline
\end{tabular}

$P 1$ :Blank vs Mock P2:Blank vs NC P3:Blank vs si si囚sortilin knockdown group

Table \. The Ct of p53LNFKB and MMP9 in each group after knocking down sortilin in Capan1 cells 


\begin{tabular}{llllll} 
& & Blank & NC & Mock & si \\
Capan1 & P53 & $20.568 \pm 0.759$ & $20.658 \pm 0.36$ & $20.703 \pm 0.487$ & $21.601 \pm 0.401$ \\
\cline { 2 - 5 } & GAPDH & $14.513 \pm 0.291$ & $14.51 \pm 1.496$ & $14.594 \pm 1.54$ & $15.996 \pm 0.545$ \\
\hline $2^{-\triangle \Delta C T}$ & $1.003 \pm 0.086$ & $1.042 \pm 0.166$ & $1.071 \pm 0.179$ & $1.517 \pm 0.225$ \\
\hline Increase rate & & $3.90 \%$ & $6.70 \%$ & $56.40 \%$ \\
\hline$P$ & & 0.474 & 0.97 & 0.002 \\
\hline NFKB & $23.135 \pm 1.063$ & $22.943 \pm 0.545$ & $23.259 \pm 0.049$ & $25.105 \pm 0.299$ \\
\hline GAPDH & $14.49 \pm 0.237$ & $14.352 \pm 1.413$ & $14.713 \pm 0.867$ & $15.86 \pm 0.81$ \\
\hline $2^{-\triangle \triangle C T}$ & $1.001 \pm 0.037$ & $1.041 \pm 0.08$ & $1.072 \pm 0.048$ & $0.67 \pm 0.129$ \\
\hline Inhibition rate & & $-0.04 \%$ & $-7.20 \%$ & $33 \%$ \\
\hline$P$ & & 0.095 & 0.217 & 0.002 \\
\hline MMP9 & $32.142 \pm 0.147$ & $31.218 \pm 0.165$ & $32.522 \pm 0.11$ & $34.547 \pm 0.09$ \\
\hline GAPDH & $14.866 \pm 0.07$ & $13.977 \pm 0.083$ & $15.22 \pm 0.214$ & $15.738 \pm 0.131$ \\
\hline $2^{-\triangle \triangle C t}$ & $1.008 \pm 0.155$ & $1.031 \pm 0.145$ & $0.984 \pm 0.066$ & $0.351 \pm 0.073$ \\
\hline Inhibition rate & & $-2.30 \%$ & $0.16 \%$ & $64.90 \%$ \\
\hline$P$ & & 0.4 & 0.6 & 0.0001
\end{tabular}

Table $\mathbb{~}$. The Ct of p53LNFKB and MMP9 in each group after knocking down sortilin in Bxpc3 cells

\begin{tabular}{llllll} 
& & Blank & NC & Mock & si \\
\hline Bxpc3 & P53 & $24.356 \pm 1.597$ & $22.546 \pm 1.117$ & $23.505 \pm 0.605$ & $21.124 \pm 0.256$ \\
\cline { 2 - 5 } GAPDH & $16.907 \pm 0.881$ & $15.01 \pm 0.464$ & $16.048 \pm 1.33$ & $14.042 \pm 0.61$ \\
\hline $2^{-\triangle \triangle C T}$ & $1 \pm 0.034$ & $1.027 \pm 0.101$ & $1.083 \pm 0.085$ & $1.407 \pm 0.137$ \\
\hline Increase rate & & $2.70 \%$ & $8.30 \%$ & $40.70 \%$ \\
\hline$P$ & & 0.351 & 0.101 & 0.006 \\
\hline NFKB & $23.991 \pm 1.797$ & $23.181 \pm 0.619$ & $23.954 \pm 0.949$ & $22.259 \pm 0.124$ \\
\hline GAPDH & $16.899 \pm 0.986$ & $15.864 \pm 0.21$ & $16.686 \pm 1.828$ & $14.326 \pm 0.835$ \\
\hline $2^{-\triangle \triangle C T}$ & $1 \pm 0.014$ & $0.924 \pm 0.076$ & $0.957 \pm 0.071$ & $0.603 \pm 0.034$ \\
\hline Inhibition rate & & $7.60 \%$ & $4.30 \%$ & $39.70 \%$ \\
\hline$P$ & & 0.083 & 0.116 & 0.0001 \\
\hline MMP9 & $31.041 \pm 1.797$ & $31.427 \pm 0.619$ & $29.936 \pm 0.949$ & $39.512 \pm 0.124$ \\
\hline GAPDH & $13.711 \pm 0.986$ & $14.09 \pm 0.21$ & $12.761 \pm 1.828$ & $17.011 \pm 0.835$ \\
\hline $2^{-\triangle \triangle C C t}$ & $1.004 \pm 0.014$ & $0.972 \pm 0.076$ & $1.084 \pm 0.071$ & $0.027 \pm 0.034$ \\
\hline Inhibition rate & & $2.90 \%$ & $-8.40 \%$ & $97.30 \%$ \\
\hline$P$ & & 0.896 & 0.143 & 0.002 \\
\hline
\end{tabular}




\section{Figures}
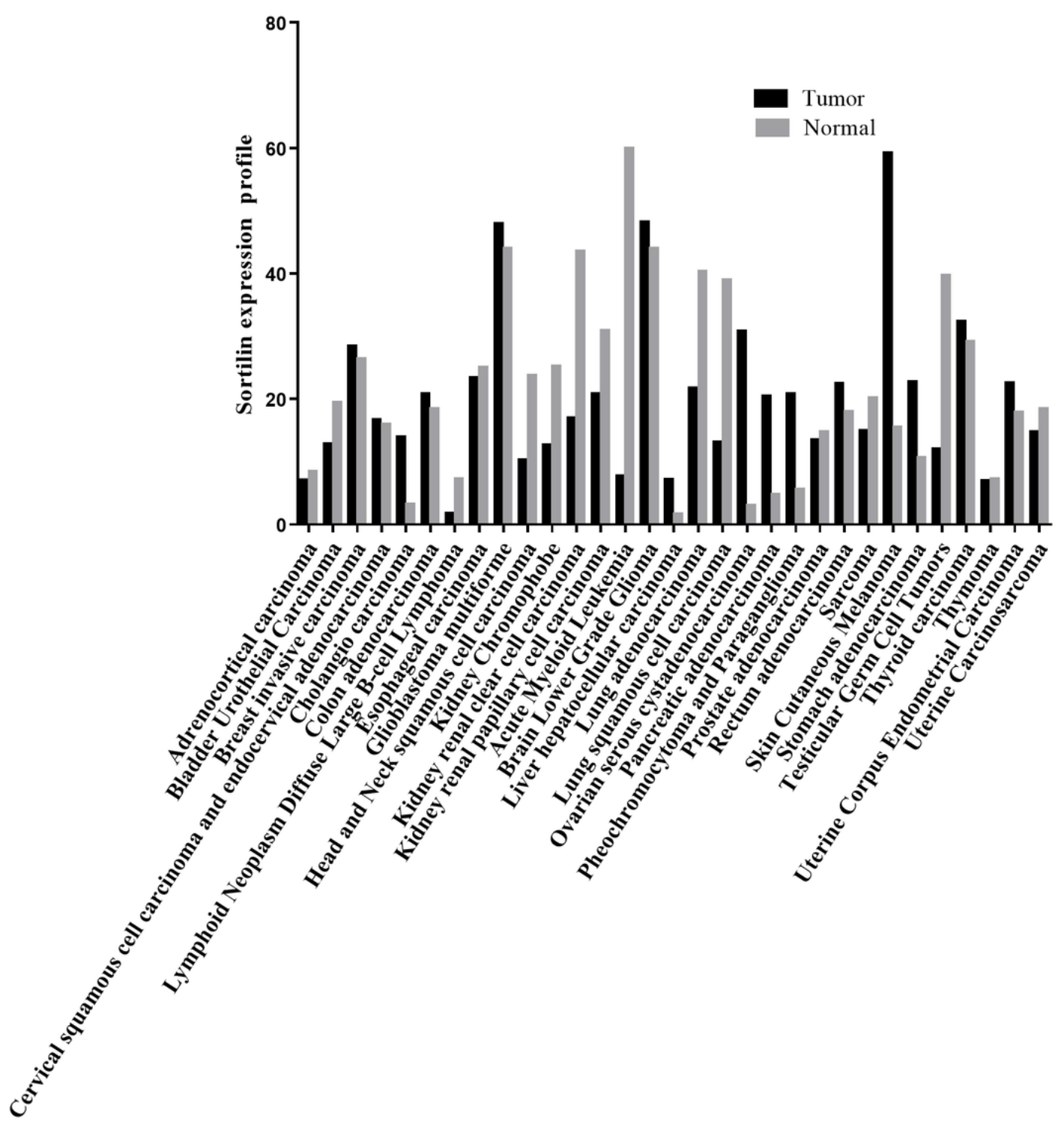

Figure 1

Legend not included with this version 


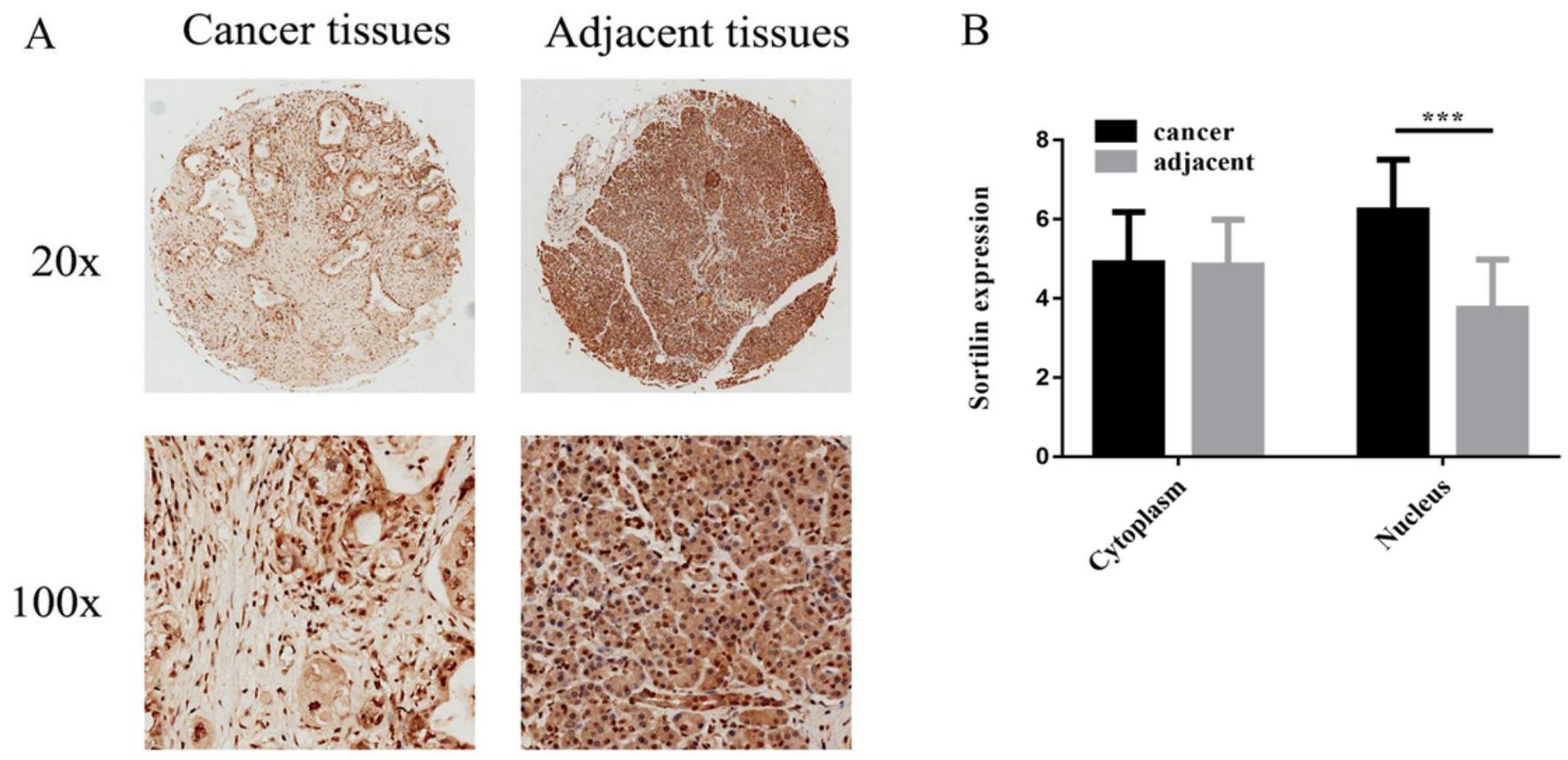

Figure 2

Legend not included with this version 


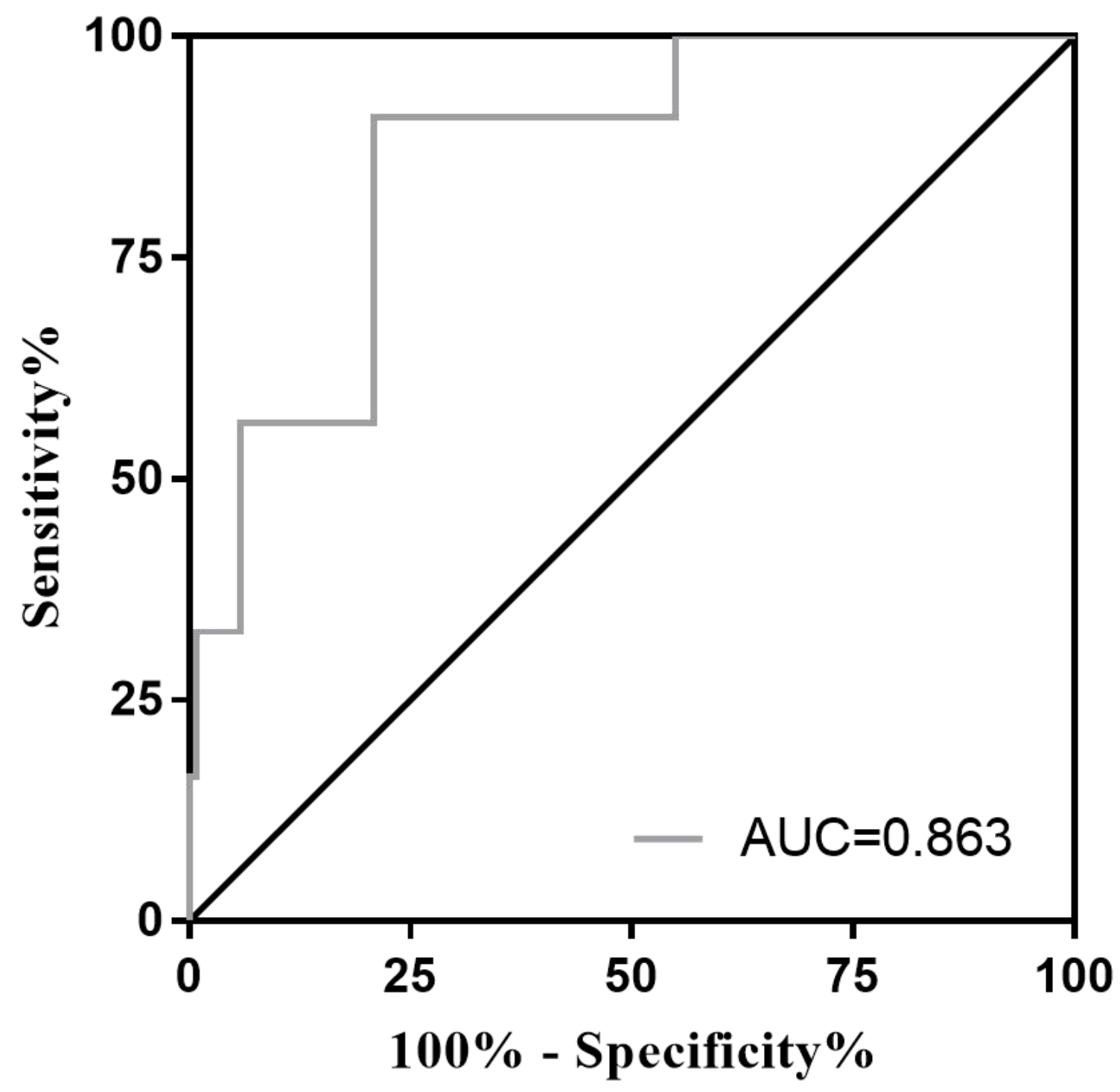

Figure 3

Legend not included with this version 


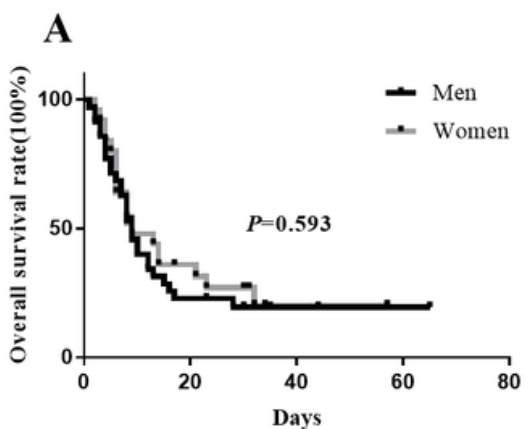

\section{D}

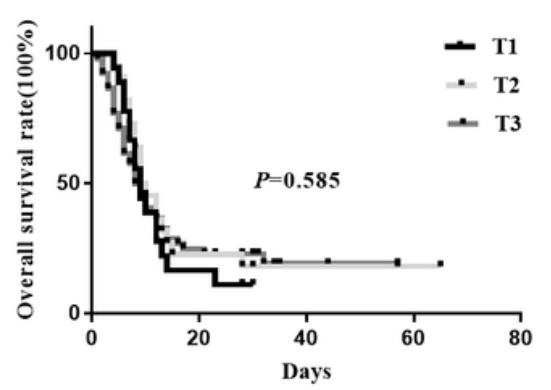

G

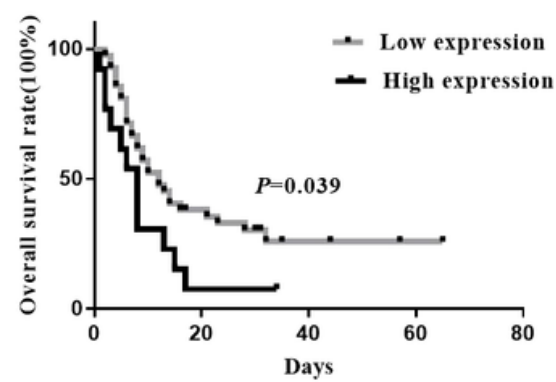

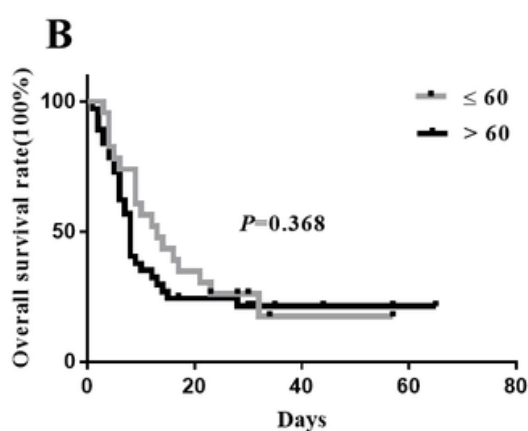

E

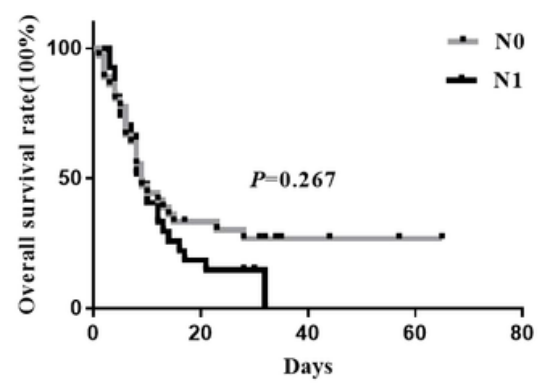

H

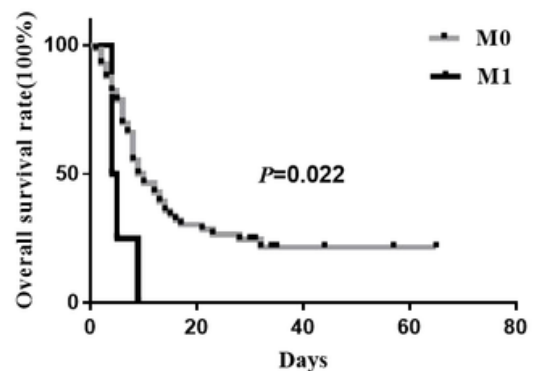

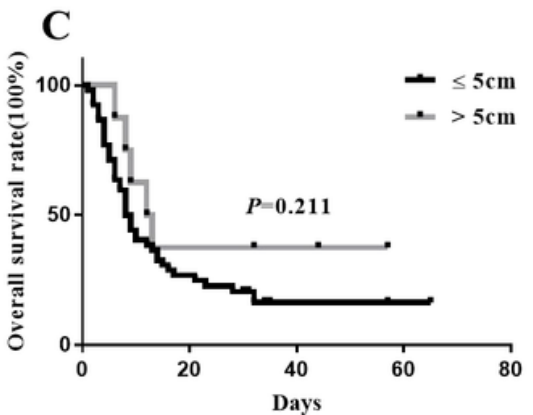

\section{F}

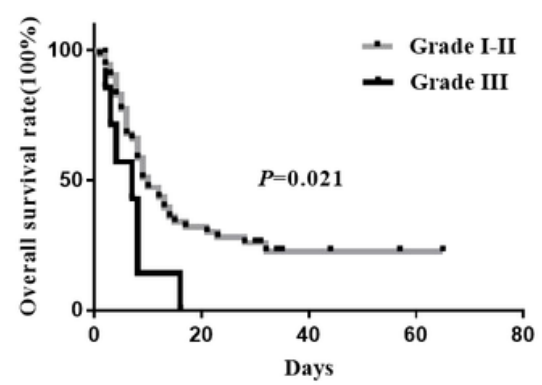

I

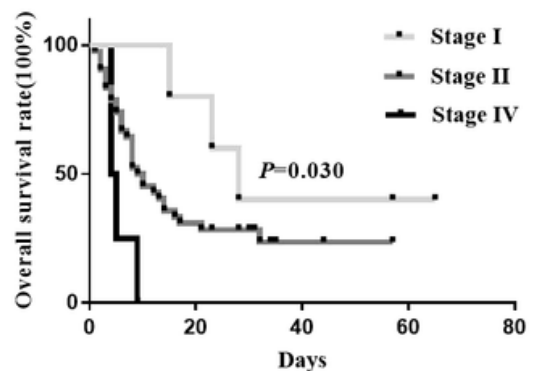

Figure 4

Legend not included with this version 


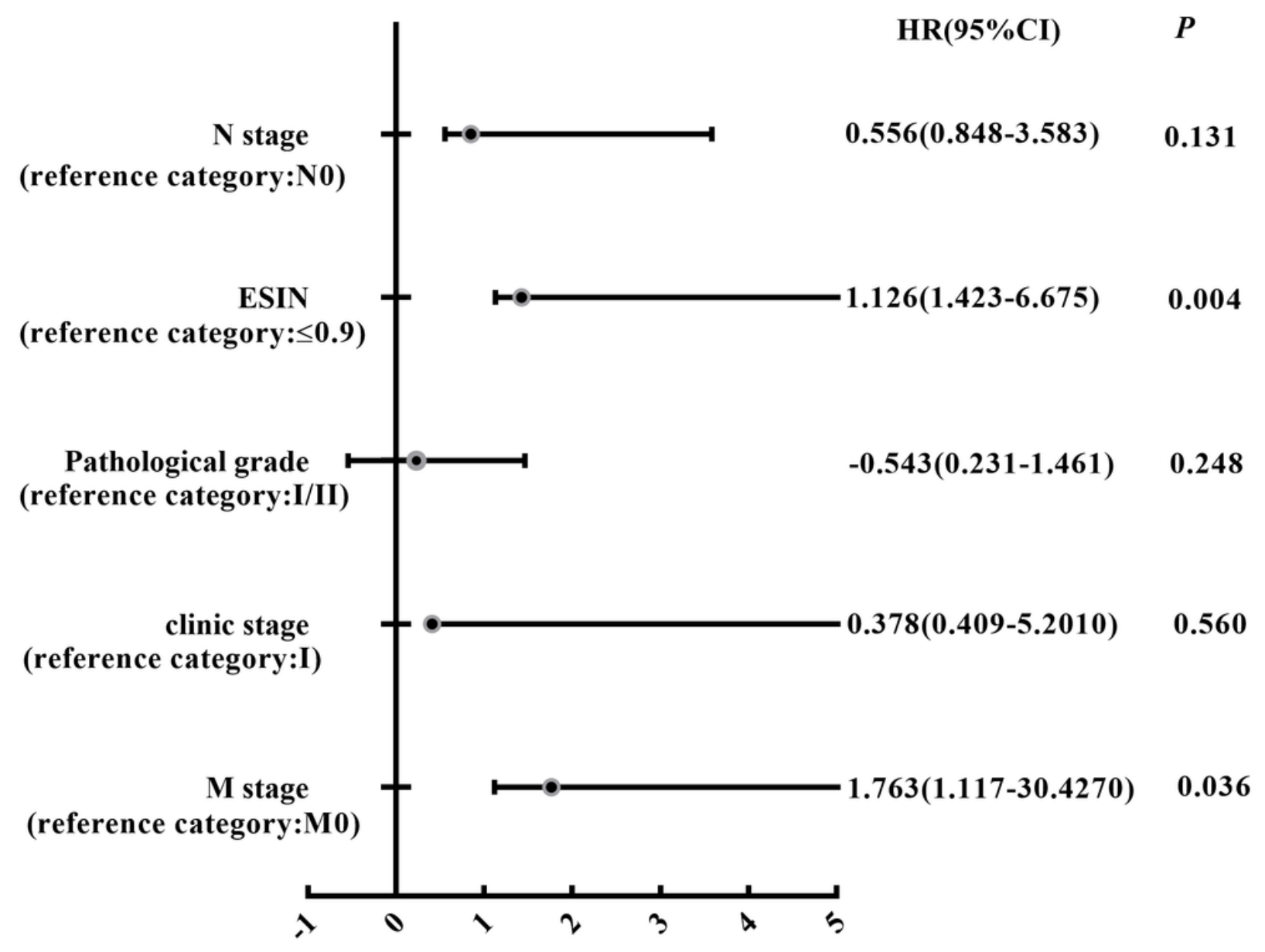

Figure 5

Legend not included with this version 


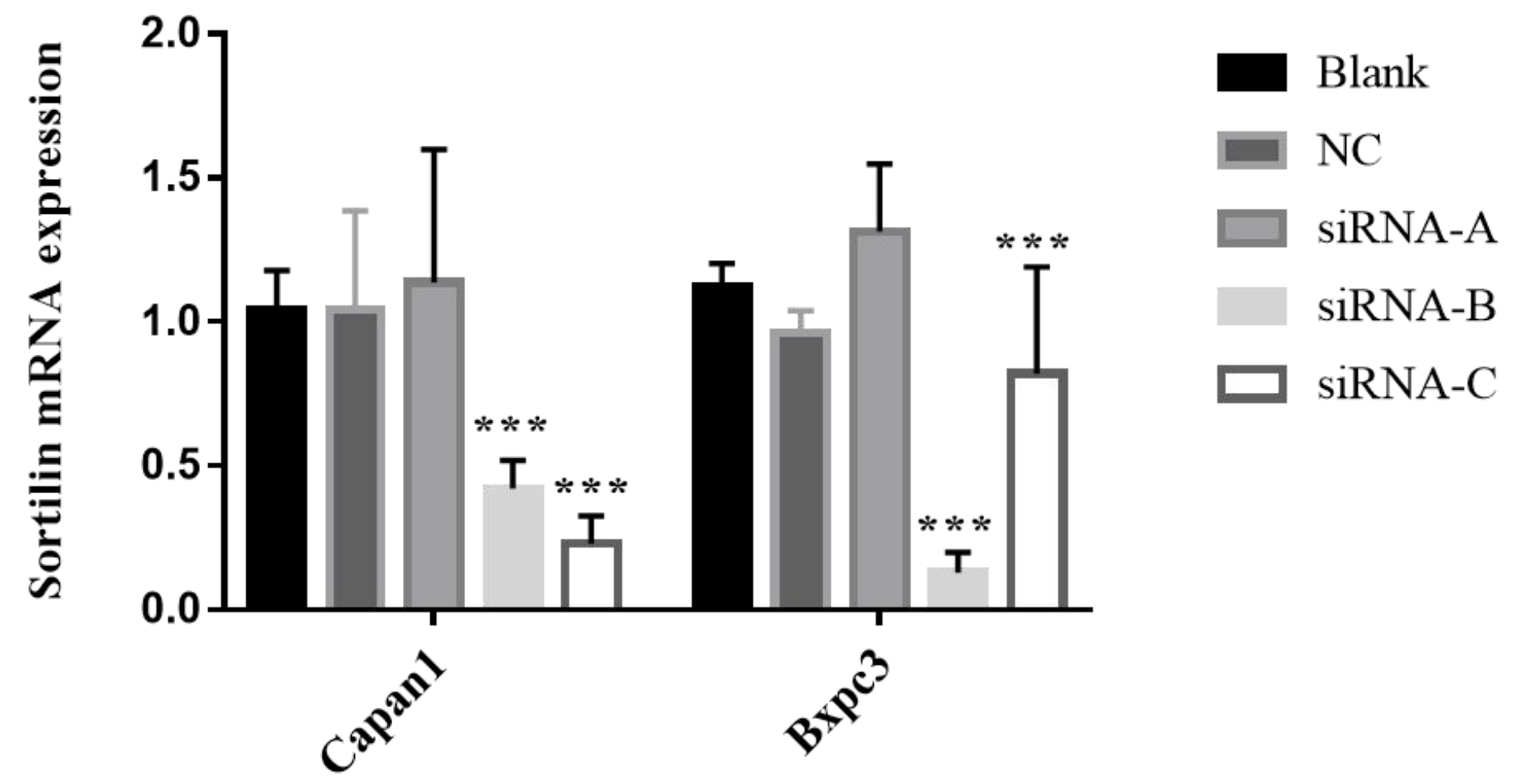

Figure 6

Legend not included with this version 


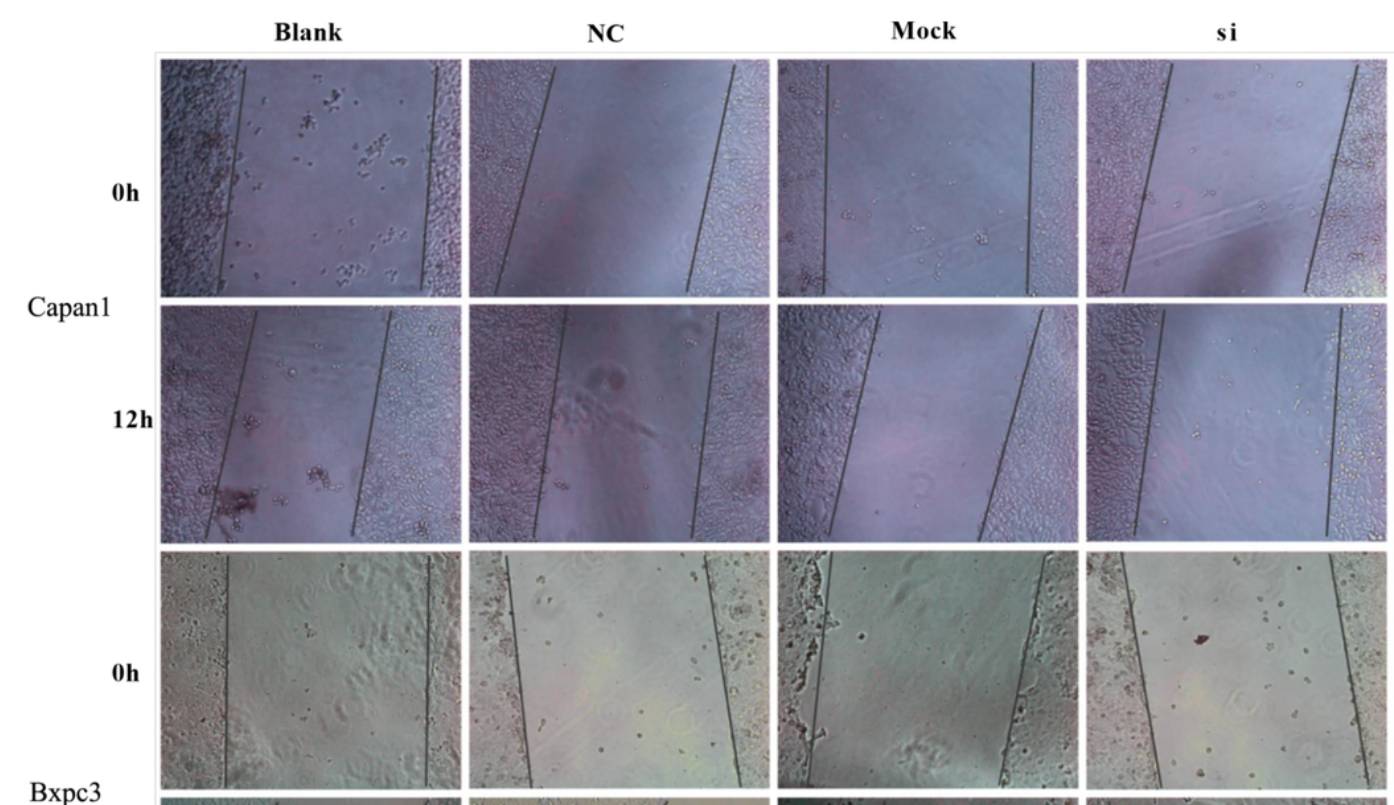

Bxpc3
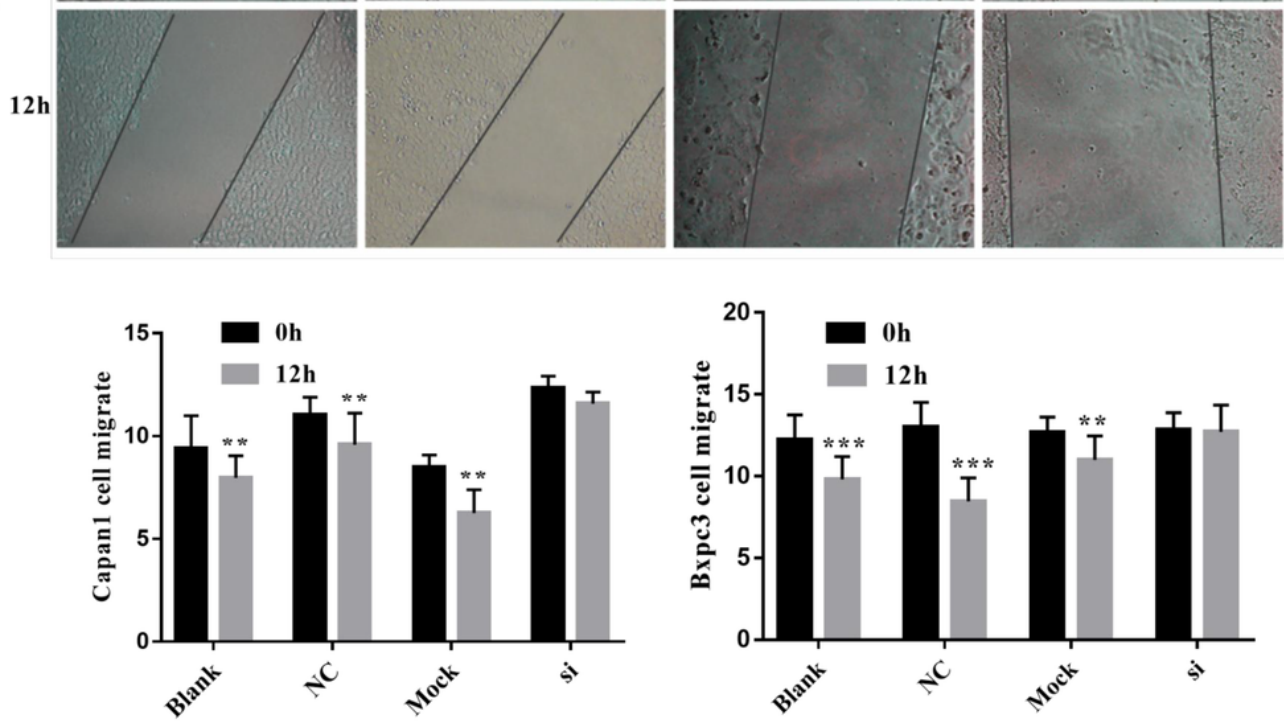

Figure 7

Legend not included with this version 


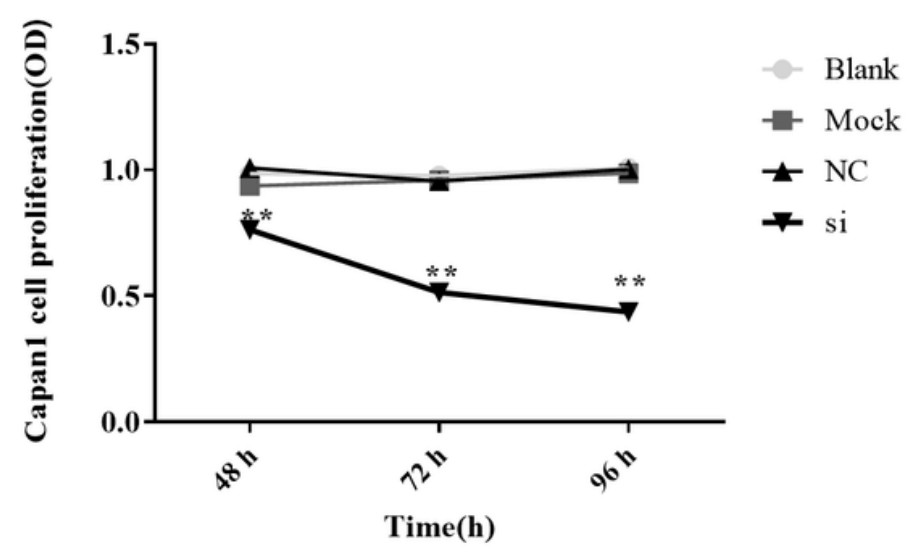

A

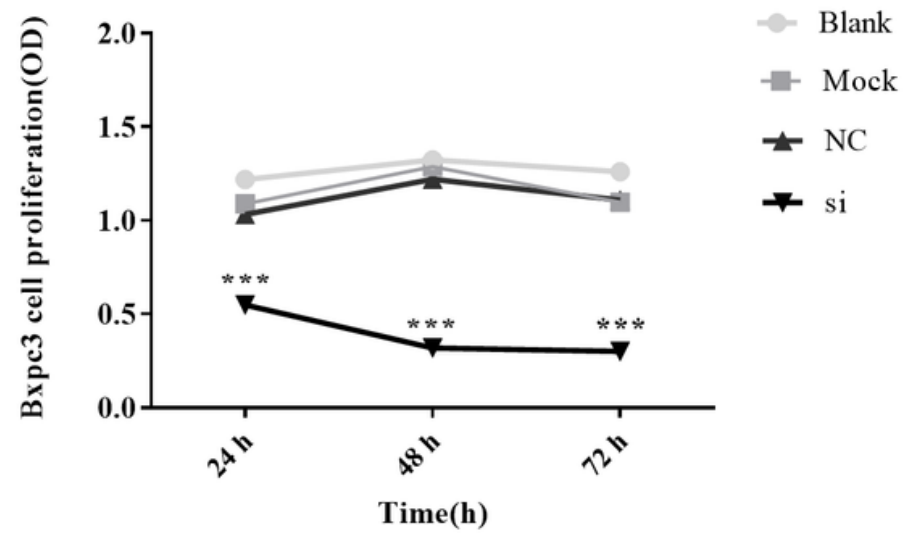

B

Figure 8

Legend not included with this version
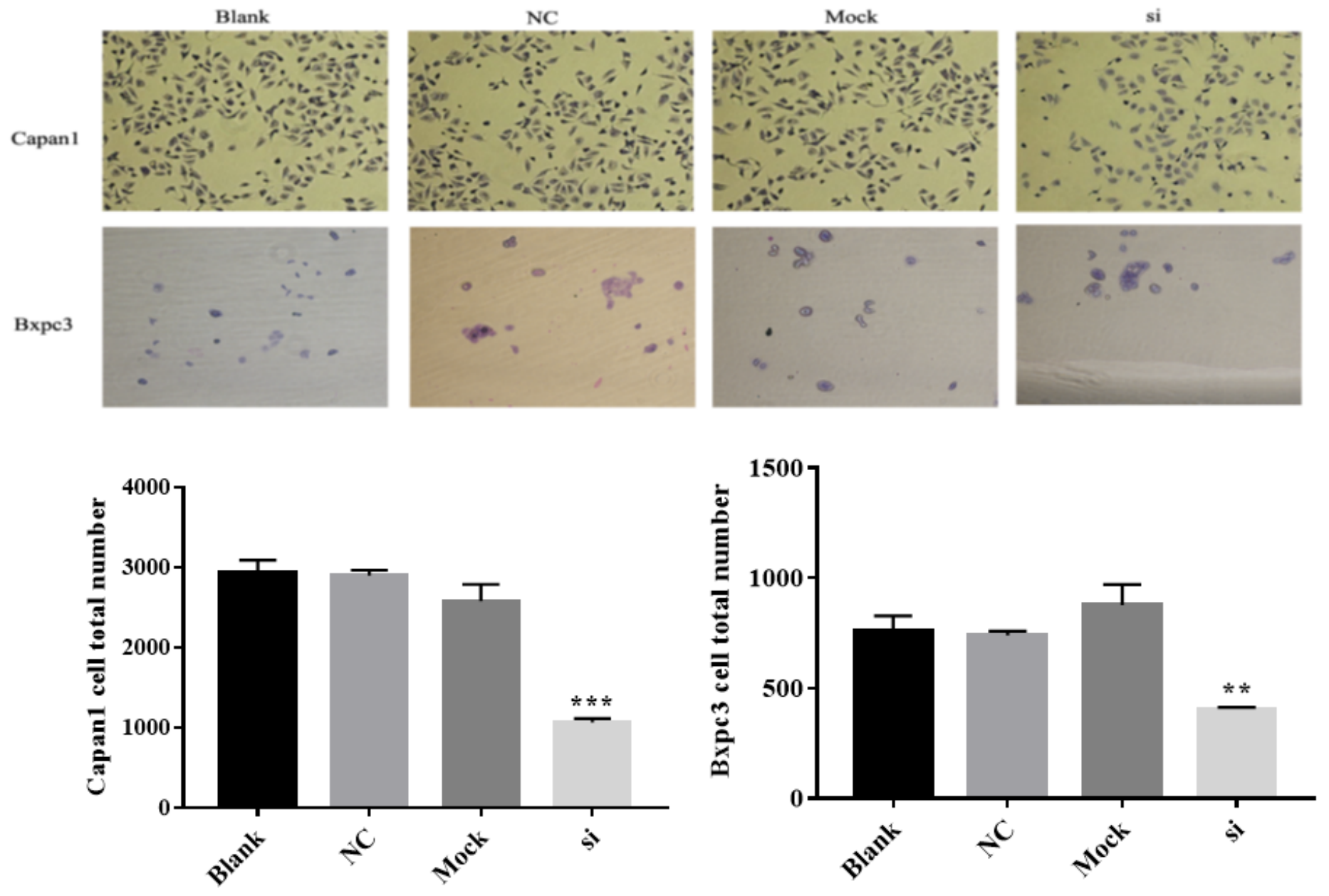

Figure 9 


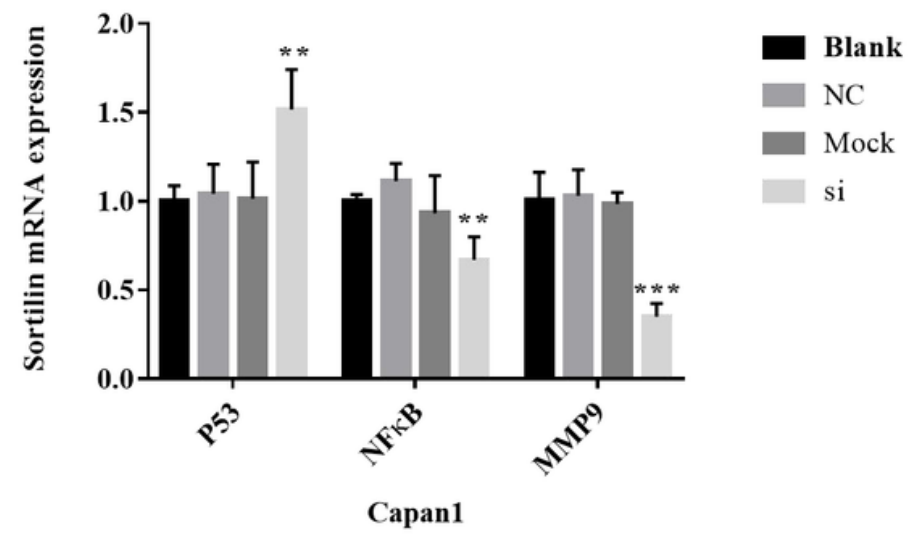

A

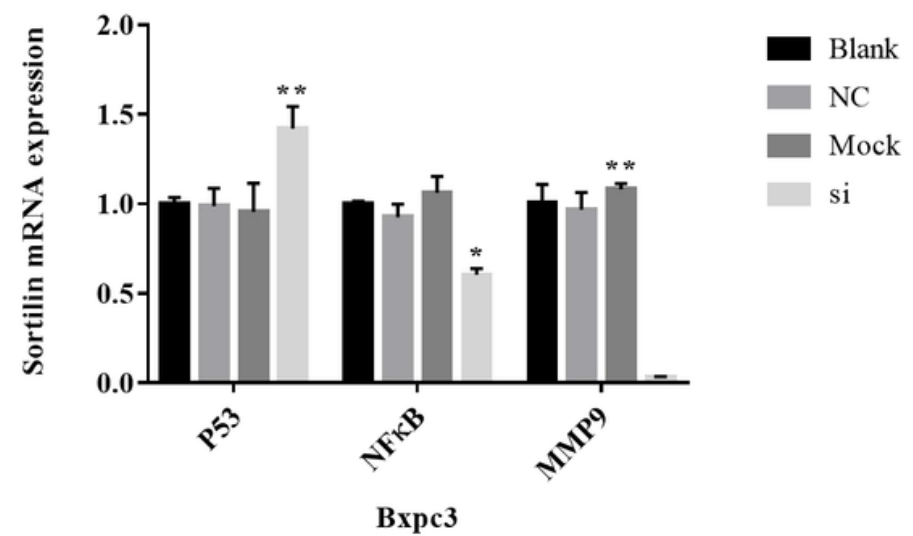

B

Figure 10

Legend not included with this version 